\title{
Eduardo Mallea: Suplemento a una Bibliografía
}

$\mathrm{D}$ ESDE la aparición de Historia de una pasión argentinal en I937, se ha considerado a Mallea uno de los más destacados intérpretes de los ideales argentinos. Sus novelas, tan cargadas de su propia personalidad, son un análisis del alma argentina, vista desde un punto de vista espiritual que llega a tener resonancias místicas.

El incremento considerable de referencias sobre la obra de Mallea exige una guía bibliográfica. Esta ha sido precisamente la labor hecha por el señor Horacio Jorge Becco, cuya valiosa contribución aparece en el volumen I de las Obras completas de Mallea.

La presente lista pone al día la parte V (Crítica sobre su obra), pues incluye el año $\mathrm{I}_{96} 6_{3}$ como también otras referencias anteriores a I96r que el señor Becco no examinó.

Este suplemento representa una revisión de más de treinta revistas, como también otras fuentes bibliográficas, guías e índices de una clase u otra.

University of Wisconsin

Marjorie J. Pinkerton

Aldrich, Earl M., "La razón humana", Handbook of Latin American Studies, No 24 (cI962), 298.

- "Las travesías", Handbook of Latin American Studies, No 24 (crg62), 298.

1 Eduardo Mallea, Historia de una pasión argentina (Buenos Aires: Sur, 1937).

2 Mallea, Obras completas (Buenos Aires: Emecé, 1961). 
Alonso Pedraz, Martín, Evolución sintáctica del español. Madrid: Aguilar, I962, pp. 389-390.

A [moroso], V[incenzo], Eduardo Mallea: "Tutto il verde perirá", Quaderni Ibero-Americani, III (dic. 1956), Pp. 27r-272.

Anderson, Robert W., "The Bay of Silence", Atlantic Monthly, I73 (May I944), pp. I3I-I33.

Balbini, José, "Historia de una pasión argentina", Universidad (Santa $\mathrm{Fe}$ ), IV (julio r938), pp. I65-I67.

Baeza Flores, Alberto, Eduardo Mallea: "La vida blanca", Cuadernos, 55 (diciembre I96I), pp. 88-89.

Baralis, Márta, Eduardo Mallea: "Coherencia de una vocación", Universidad (Santa Fe), 55 (enero-marzo 1963), pp. 75-83.

Brittan, Jeanne Mann, "The Bay of Silence", Library Journal, 69 (marzo I, I944), p. 204.

Bueno, Salvador, "Pensamiento y pasión de Eduardo Mallea", Revista (Federación de Doctores en Ciencias y en Filosofía, La Habana), (junio-diciembre I945), Pp. I40-I5I.

Sobre Historia de una pasión argentina.

Canal Feijoo, Bernardo. Eduardo Mallea: "La vida blanca", Sur, № 269 (marzo-abril I961), pp. 68-79.

Castillo, Homero, "Posesión", Books Abroad, XXXIV (Spring, 1960), p. I66.

Cruz, Jorge. "La representación de los aficionados" de Eduardo Mallea, Cuadernos, 73 (junio 1963 ), pp. 84-85.

Dauster, Frank. "El gajo de enebro", tragedia en tres actos; Handbook of Latin American Studies, № 21 (cr959), p. 227.

—, "Las travesías [I]", Revista Hispánica Moderna, XXIX (enero rg63), p. 72.

Debenedetti, Edith, Horacio Pereyra, "El gobierno de Mallea y el intento revolucionario de Juan Antonio Rodríguez", Trabajos y Comunicaciones (Instituto de Investigaciones Históricas, Universidad Nacional de La Plata), X (I96r), pp. 63-8x.

Erro, Carlos Alberto, Eduardo Mallea: "Las travesías", Nación (I4 de enero, I962).

F., A. B., "La vida blanca", Cuadernos, No 55 (I96I), pp. 88-99.

Ferrándiz Alborz, Francisco, "Historia de una pasión argentina", Cuddernos Americanos, № I I8 (septiembre-octubre I96I), pp. 231-248. Flores, Angel, "The Bay of Silence", Book Week (Cbicago Sun), (March 19, 1944), P. 9. 
-. "Notas de un novelista", Handbook of Latin American Studìes, No. I9 (1957), p. 252.

Gallo, Ugo, Storia della letteratura ispano-americana, [Di] Ugo Gallo [e] Giuseppe Bellini, 2nd ed. Milano: Nuovo Accademia, I958. pp. 399-400.

Gibbs, Beverly Jean, "Spatial Treatment in the Contemporary Psychological Novel of Argentina", Hispania (AATSP), XLV (1962), pp. 4ro$4 \mathrm{I} 4$.

—-, "A Study of Five Contemporary Psychological Novels of Argentina", Unpublished $\mathrm{Ph}$. D. dissertation. University of Wisconsin, Madison, I960, pp. I86-224.

Estudio de siete caracteres de La sala de espera.

Giménez Pastor, Arturo, Historia de la literatura argentina, Buenos Aires: Labor, 1945, p. 556.

Gómez, Carlos Alberto, "Mallea de siempre", Gaceta (Tưumán), No I7 (3 de julio 1960), p. 627.

Sobre La razón humana.

Grieben, Carlos F., Eduardo Malled, Buenos Aires: Ediciones Culturales Argentinas, I96r, I29 pp.

(Biblioteca del Sesquicentenario. Serie Argentina de Letras).

Henríquez Ureña, Pedro, Las corrientes literarias en la América Hispánica, México: Fondo de Cultura Económica, r949, p. 203.

- Historia de la cultura en la América Hispánica, México: Fondo de Cultura Económica, 1947, p. 165.

Hermes Villordo, Oscar, Eduardo Mallea: "La representación de los aficionados", Sur, N2 282 (mayo-junio I963), pp. 89-9r.

Hernández Arregui, Juan José, "Jorge Luis Borges y Eduardo Mallea", Nuestro Tiempo (La Habana), V (septiembre-octubre 1958), pp. 9-I 2 , port.

Justo, Luis, "Mallea, o la literatura como sustento de la realidad", Sur, No 280 (enero-febrero x $^{6} 3$ ), pp. $5^{\text {I-56. }}$

Sobre Las travesías, tomos I y 2.

Kirstein, Lincoln. "Recent Latin American Novels", New Republic, I ro (April 24, 1944), 574-575.

La Cruz, Salvador de, "Nuevos novelistas iberoamericanos", El Libro y el Pueblo (México), XVII (marzo, r955), pp. 5-75, port.

Lancelotti, Mario A., "Notas de un novelista", Sur, No 232 (enero-febrero I955), Pp. 37-39.

Lewald, A. Ernest," "An Introduction to the Literature on Buenos Aires 
and Its Inhabitants", Modern Language Journal, XLV (196r), p. I63.

Lichtblau, Myron I., "El concepto del tiempo en las obras de Eduardo Mallea", Humanitas (Monterrey), № 3 (I962), pp. 299-314.

—, Eduardo Mallea: "Posesión", Revista Iberoamericana, XXV (julio-diciembre 1960), Pp. 348-350.

- Some Considerations on the Structure of Eduardo Mallea's "La bahía de silencio", Symposium (Syracuse), XV (I96I), pp. 204-2I3.

—, "Temas y técnica en los cuentos de Eduardo Mallea", Humanitas (Monterrey), II (I96r), pp. 389-406.

Marongoni, Víctor, "Eduardo Mallea, novelista", Estudios (Buenos Aires), 50 (septiembre 1961), pp. 525-533.

Morsella, Astur, "Revisión de Mallea", in "La literatura argentina", in Gaceta Literaria (Buenos Aires), IV (mayo ig60).

Neale-Silva, Eduardo, "Chaves", Handbook of Latin American Studies, No I9 (cr957), p. 260.

-. "La sala de espera", Handbook of Latin American Studies, № I9 (cr957), p. 260.

—, "Simbad", Hand'book of Latin American Studies, No 2 (x959), p. 209.

Neish, A., "Letter from Buenos Aires", Nation, I96 (March 30, 1963), p. 273.

Pagés Larraya, Antonio, "Mirada a la novela", Ficción, Nos. 24-25 (marzo-junio 1960), pp. 27-36.

Paita, Jorge A., "Dos obras de Eduardo Mallea", Surr, № 248 (septiembreoctubre r957), pp. 87-94.

Sobre Simbad y El gajo de enebro.

Peltzer, Federico J., "Algunas corrientes dentro de la narración argentina contemporánea", Estudios (Buenos Aires), 50 (diciembre r96r), pp. $797-802$.

Petersen, Fred, "Notes on Mallea's Definition of Argentina", Hispania (AATSP). XLV (December. I962). pp. 621-624.

Picón-Salas, Mariano, "Prólogo a Mallea", in Crisis, cambio tradición... Caracas. Editorial Edime, 1955.

Ríos Patrón, José Luis, "El estilo de Mallea", Biblioteca (Buenos Aires), IX (I957), pp. 6r-87.

Rodríguez-Alcalá, Hugo, "Eduardo Mallea: Posesión", Revista Hispánica Moderna, XXVI (1960) p. I27.

[Roggiano, Alfredo A.], ["Eduardo Mallea"] in Pan American Union. 
Letters Section. Diccionario de la literatura bispanoamericana [v.4] Argentina, Segunda Parte, Washington, D. C.: Unión Panamericana, I961, pp. 319-323.

Estudio bioblibliográfico.

Silvetti Paz, N., Eduardo Mallea: "Posesión", Sur, No 260 (septiembre/ octubre. I959), pp. $77-83$.

Trilling, Diana, "Fiction in Review", Nalion, I58 (April 15, 1944), Pp. 454-455.

Torre, Guillermo de, "Plenitud de Eduardo Mallea", Nación, (23 de septiembre 1962).

Sobre Obras completas, I

—, "Plenitud de Eduardo Mallea", Cuddernos, № 66 (noviembre I962), pp. 89-9I.

Sobre Obras completas; $\mathrm{r}$.

Ulloa, María Elisa, "Mallea's Argentina", Americas, VII (March 1955), pp. 28-32.

La edición inglesa del artículo que aparece en la edición española de Américas de abril de 1955.

Undurraga, Antonio de, Eduardo Mallea: "La razón humana", Cuadernos, $\mathrm{N}^{\circ} 48$ (mayo 1961), p. 88.

Williams Alzaga, Entique, La pampa en la novela argentina, $\mathrm{I}^{\mathrm{a}}$ ed. Buenos Aires: A. Estrada, r955.

Incluye Mallea; v. Handbook of Latin American Studies, № 20 (cr958), p. 248.

Wolfe, Bertram D., Argentine Portraits: "Mr. Mallea Writes of the Tragic Dilemmas Facing the Man of Good Will in Our Time", New York Herald Tribune (March 26, 1944), p. 4.

Sobre The Bay of Silence.

Zum Felde, Alberto, "Eduardo Mallea y la búsqueda apasionada de la argentinidad", in Vol. x: Los ensayistas, of his Indice crítico de la literatura bispanoamericana, 2 v. México: Ed. Guarania, 1954, pp. 455-46r. 
